\title{
Asymptotic behavior of eigenvalues of hydrogen atom equation
}

\author{
Etibar S Panakhov and Ismail Ulusoy ${ }^{2 *}$
}

\section{"Correspondence:}

iulusoy@adiyaman.edu.tr

${ }^{2}$ Department of Mathematics,

Adiyaman University, Adiyaman,

02040, Turkey

Full list of author information is

available at the end of the article

\section{Introduction}

The distribution of eigenvalues in differential operator's spectral theory has an important place. This classic issue was first examined in a finite interval for second order operators in the 19th century by Sturm and Liouville. Later, where the regular boundary conditions were satisfied, the distribution of eigenvalues of differential operators in a finite interval in arbitrary order was also examined by Birkhoff in 1908 [1].

Especially, the distribution of eigenvalues of the operators with a discrete spectrum defined in the whole of space for quantum mechanics has great importance. Firstly, the formula for the distribution of the eigenvalues of the single-dimensional Sturm operator defined in the whole of the straight-line axis with increasing potential at infinity was given by Titchmarsh in 1946 [2]. Titchmarsh also has shown the distribution formula for the Schrödinger operator. In later years, Levitan and Gasymov improved the Titchmarsh method and found important asymptotic formulas for the eigenvalues of different differential operators [3, 4].

Two important methods have been dealt with to examine the asymptotic formula for eigenvalues. The first method, the variation method, is due to Courant and Hilbert [5]. Birman and Solomyak have improved this method in recent years [6]. The second method that is related with the resolvent of the operator in question was suggested by Carleman [7]. Another important method for examining the asymptotic of the eigenvalues in singular condition was suggested by Fedoryuk [8]. This method is very useful in that it ensures that the distribution of the eigenvalues of the operators with partial derivation are such that the coefficients are analytic functions. Later, many studies have been conducted to examine the eigenvalues [9-25]. Many mathematicians have examined the eigenvalues so far.

The spectral problem for the Sturm-Liouville operator with Dirichlet boundary condition is given in detail in [9] by Poeschel and Trubowitz. Guillot and Ralston have extended 
these results to the singular Sturm-Liouville operator

$$
L=-\frac{d^{2}}{d x^{2}}+\frac{2}{x^{2}}+q(x)
$$

with domain $\left\{y \in L^{2}[0,1]: y, y^{\prime}\right.$, absolutely continuous on $(0,1], L y \in L^{2}[0,1]$ and $\left.y(1)=0\right\}$ [16].

Later, this work was generalized by Carlson [10]. For real numbers $b$ and real valued functions $q(x) \in L^{2}[0,1]$, Carlson dealt with the operator

$$
L(m, q)=-\frac{d^{2}}{d x^{2}}+\frac{m(m+1)}{x^{2}}+q(x), \quad m=0,1,2, \ldots
$$

with domain $\left\{y \in L^{2}[0,1]: y, y^{\prime}\right.$, absolutely continuous on $(0,1], L(m, q) y \in L^{2}[0,1]$, $\lim _{x \downarrow 0} y(x)=0$ and $\left.y^{\prime}(1)+b y(1)=0\right\}$. Similar features of the Sturm-Liouville operator were studied in [16-18].

Consider the Schrödinger equation for two particles in dimensionless variables,

$$
-\frac{\partial^{2} \psi}{\partial x^{2}}-\frac{\partial^{2} \psi}{\partial y^{2}}-\frac{\partial^{2} \psi}{\partial z^{2}}+V(x, y, z) \psi=k^{2} \psi
$$

If the potential function $V(x, y, z)$ depends only on $r=\left(x^{2}+y^{2}+z^{2}\right)^{1 / 2}$, i.e. $V(x, y, z)=V(r)$, then the variables in (1.1) can be separated by putting

$$
\psi(x, y, z)=\frac{\psi(r)}{r^{1 / 2}} Y_{m}^{l}(\theta, \varphi), \quad l=0,1,2, \ldots
$$

where $x=r \sin \theta \cos \varphi, y=r \sin \theta \sin \psi, z=r \cos \theta$, and $Y_{m}^{l}(\theta, \varphi)$ are the spherical harmonics. This gives a differential equation of the form

$$
\frac{d^{2} \varphi}{\partial r^{2}}+\frac{1}{r} \frac{d \varphi}{\partial r}-\frac{\lambda^{2}}{r^{2}}-V(r) \varphi+k^{2} \varphi=0
$$

for the function $\varphi(r)$, where $\lambda=l+1 / 2(l=0,1,2, \ldots)$. If the potential function $V(r)$ satisfies the condition $\int_{0}^{\infty} r|V(r)| d r<\infty$, then, for a solution of (1.2), which is regular at zero and normalized, the following asymptotic formula is satisfied:

$$
r^{1 / 2} \varphi(r, k, \lambda)=A(k, \lambda) \sin \left[k r-\frac{\pi}{2}\left(\lambda-\frac{1}{2}\right)+\delta(k, \lambda)\right]+o(1)
$$

for fixed $\lambda$, and $k$, and $r \rightarrow \infty$.

In this formula, $A(k, \lambda)$ is called the scattering amplitude and $\delta(k, \lambda)$ the scattering phase or phase shift [4].

In quantum mechanics the study of the energy levels of the hydrogen atom leads to the equation [26-28]

$$
-\frac{d^{2} R}{d r^{2}}+\frac{a}{r} \frac{d R}{d r}-\frac{l(l+1)}{r^{2}} R+\left(E+\frac{a}{r}\right) R=0 \quad(0<r<\infty) .
$$

The substitution $R=y / r$ reduces this equation to the form

$$
y^{\prime \prime}+\left[E+\frac{2}{r}-\frac{l(l+1)}{r^{2}}\right] y=0 .
$$


Our aim here is to find the asymptotic behavior of the eigenvalues of the problem

$$
\begin{aligned}
& -y^{\prime \prime}+\left[\frac{2}{x^{2}}-\frac{2}{x}+q(x)\right] y=\lambda y \quad(0<x \leq 1), \\
& y(1)=0
\end{aligned}
$$

with domain $\left\{y \in L^{2}[0,1]: y, y^{\prime}\right.$ that are absolutely continuous on $\left.(0,1], L y \in L^{2}[0,1]\right\}$. Here we have $\lambda=\sqrt{-E}, E<0$.

Spectral problems for the hydrogen atom equation were considered by many mathematicians. Particularly, the inverse problem was examined in Panakhov and Yilmazer's papers $[12,13]$.

\section{Basic properties}

We consider the singular Sturm-Liouville equation

$$
-y^{\prime \prime}+\left[\frac{2}{x^{2}}-\frac{2}{x}+q(x)\right] y=\lambda y, \quad x \in(0,1]
$$

where the function $q(x) \in L^{2}[0,1]$. Let us give the solutions of this equation by integral equation representations.

Lemma 1 The solutions of (2.1) have the following form:

$$
\varphi(x, \lambda, q)=x^{2}+\frac{1}{3} \int_{0}^{x}\left(\frac{x^{2}}{t}-\frac{t^{2}}{x}\right)\left(q(t)-\frac{2}{t}-\lambda\right) \varphi(t, \lambda, q) d t
$$

and

$$
\psi(x, \lambda, q)=c_{1} x^{2}+c_{2} x^{-1}-\frac{1}{3} \int_{x}^{1}\left(\frac{x^{2}}{t}-\frac{t^{2}}{x}\right)\left(q(t)-\frac{2}{t}-\lambda\right) \psi(t, \lambda, q) d t
$$

where $q(x) \in L^{2}[0,1]$.

Proof Let us show that (2.2) is satisfied. The general solution of the equation

$$
-y^{\prime \prime}+\frac{2}{x^{2}} y=0
$$

is

$$
y=c_{1} x^{2}+c_{2} x^{-1} .
$$

Let us apply the method of variation of parameters of (2.1),

$$
-y^{\prime \prime}+\frac{2}{x^{2}} y=\left[\lambda+\frac{2}{x}-q(x)\right] y .
$$

Taking the second derivative of the equation

$$
y=u_{1}(x) x^{2}+u_{2}(x) x^{-1}
$$


and substituting this into (2.1), we obtain

$$
\begin{aligned}
& u_{1}^{\prime}(x) x^{2}+u_{2}^{\prime}(x) x^{-1}=0, \\
& -2 u_{1}^{\prime}(x) x+u_{2}^{\prime}(x) x^{-2}=\left(\lambda+\frac{2}{x}-q(x)\right) y(x) .
\end{aligned}
$$

If we multiply the first equation by $-1 / x$ and combine with the second equation we have

$$
-3 u_{1}^{\prime}(x) x=\left(\lambda+\frac{2}{x}-q(x)\right) y(x)
$$

Take the integral of this equation from 0 to $x$ :

$$
u_{1}(x)=\frac{1}{3} \int_{0}^{x} \frac{1}{t}\left(q(t)-\lambda-\frac{2}{t}\right) y(t) d t
$$

If we multiply the first equation by 2 and the second equation by $x$ and combine these equations we have

$$
\frac{3}{x} u_{2}^{\prime}(x)=x\left(\lambda+\frac{2}{x}-q(x)\right) y(x)
$$

Take the integral of this equation from 0 to $x$ :

$$
u_{2}(x)=-\frac{1}{3} \int_{0}^{x} t^{2}\left(q(t)-\lambda-\frac{2}{t}\right) y(t) d t
$$

Then we get the equation

$$
y=\frac{1}{3} \int_{0}^{x}\left(\frac{x^{2}}{t}-\frac{t^{2}}{x}\right)\left(q(t)-\lambda-\frac{2}{t}\right) y(t) d t
$$

We use the above method to show (2.3). Take the integral of (2.4) from $x$ to 1 :

$$
u_{1}(x)=-\frac{1}{3} \int_{x}^{1} \frac{1}{t}\left(q(t)-\lambda-\frac{2}{t}\right) y(t) d t
$$

Take the integral of (2.5) from $x$ to 1 :

$$
u_{2}(x)=\frac{1}{3} \int_{x}^{1} t^{2}\left(q(t)-\lambda-\frac{2}{t}\right) y(t) d t
$$

Then we get the equation

$$
y=-\frac{1}{3} \int_{x}^{1}\left(\frac{x^{2}}{t}-\frac{t^{2}}{x}\right)\left(q(t)-\lambda-\frac{2}{t}\right) y(t) d t .
$$

So we proved the theorem. 
Now we will show that these solutions are analytic by using the method of successive approximations. Addressing (2.2) first, let

$$
y_{0}(x)=x^{2}, \quad y_{n+1}(x)=y_{0}(x)+\frac{1}{3} \int_{0}^{x}\left(\frac{x^{2}}{t}-\frac{t^{2}}{x}\right)\left(q(t)-\lambda-\frac{2}{t}\right) y_{n}(t) d t .
$$

Theorem 1 The sequence $y_{n}(x)$ converges uniformly to a function $\varphi(x, \lambda, q)$ satisfying $(2.2)$ and (2.1). Moreover, $\lim _{x \downarrow 0} x^{-2} \varphi(x, \lambda, q)=1$ and the mapping $(\lambda, q) \rightarrow \varphi(x, \lambda, q)$ is analytic from $\mathbb{C} \times L^{2}[0,1] \rightarrow \mathbb{C}[0,1]$.

Proof Let us show that

$$
\begin{aligned}
\left|y_{n}(x)-y_{n-1}(x)\right| \leq & x^{2}\left[\left(\frac{1}{3}\right)^{n}\left(\frac{x^{3 n}}{\prod_{m=1}^{n} 3 m}\right)^{1 / 2}\|q-\lambda\|_{2}^{n}\right. \\
& \left.+\sum_{k=1}^{n-1}\left(\frac{x^{3 n-k}}{(3 n-k) \prod_{m=1}^{n-1} 3 m}\right)^{1 / 2}\|q-\lambda\|_{2}^{n-k}+\frac{x^{n}}{n !}\right],
\end{aligned}
$$

by using the method of induction. For $k=1$,

$$
\begin{aligned}
\left|y_{1}(x)-y_{0}(x)\right| & =\left|\frac{1}{3} \int_{0}^{x}\left(\frac{x^{2}}{t}-\frac{t^{2}}{x}\right)\left(q(t)-\lambda-\frac{2}{t}\right) y_{0}(t) d t\right| \\
& =\left|\frac{1}{3} \int_{0}^{x}\left(\frac{x^{2}}{t}-\frac{t^{2}}{x}\right)\left(q(t)-\lambda-\frac{2}{t}\right) t^{2} d t\right| \\
& \leq x^{2}\left|\frac{1}{3} \int_{0}^{x}\left(t-\frac{t^{4}}{x^{3}}\right)\left(q(t)-\lambda-\frac{2}{t}\right) d t\right| .
\end{aligned}
$$

We have $\left|t-\frac{t^{4}}{x^{3}}\right| \leq t$,

$$
\begin{aligned}
\left|y_{1}(x)-y_{0}(x)\right| & \leq x^{2}\left|\frac{1}{3} \int_{0}^{x} t\left(q(t)-\lambda-\frac{2}{t}\right) d t\right| \\
& \leq x^{2}\left[\left|\frac{1}{3} \int_{0}^{x} t(q(t)-\lambda) d t\right|+\frac{2 x}{3}\right] .
\end{aligned}
$$

By the Cauchy-Schwarz inequality, we get

$$
\begin{aligned}
\left|y_{1}(x)-y_{0}(x)\right| & \leq x^{2}\left[\frac{1}{3}\left(\int_{0}^{x} t^{2} d t\right)^{1 / 2}\left(\int_{0}^{x}(q(t)-\lambda)^{2} d t\right)^{1 / 2}+\frac{2 x}{3}\right] \\
& \leq x^{2}\left[\frac{1}{3}\left(\frac{x^{3}}{3}\right)^{1 / 2}\|q-\lambda\|_{2}+x\right] .
\end{aligned}
$$

For $k=2$,

$$
\begin{aligned}
\left|y_{2}(x)-y_{1}(x)\right| & =\left|\frac{1}{3} \int_{0}^{x}\left(\frac{x^{2}}{t}-\frac{t^{2}}{x}\right)\left(q(t)-\lambda-\frac{2}{t}\right)\left(y_{1}(t)-y_{0}(t)\right) d t\right| \\
& =\left|\frac{1}{3} \int_{0}^{x}\left(\frac{x^{2}}{t}-\frac{t^{2}}{x}\right)\left(q(t)-\lambda-\frac{2}{t}\right) t^{2}\left[\frac{1}{3}\left(\frac{t^{3}}{3}\right)^{1 / 2}\|q-\lambda\|_{2}+t\right] d t\right| \\
& \leq x^{2}\left|\frac{1}{3} \int_{0}^{x}\left(t-\frac{t^{4}}{x^{3}}\right)\left(q(t)-\lambda-\frac{2}{t}\right)\left[\frac{1}{3}\left(\frac{t^{3}}{3}\right)^{1 / 2}\|q-\lambda\|_{2}+t\right] d t\right|
\end{aligned}
$$


Since $\left|t-\frac{t^{4}}{x^{3}}\right| \leq t$

$$
\begin{aligned}
\left|y_{2}(x)-y_{1}(x)\right| \leq & x^{2}\left|\frac{1}{3} \int_{0}^{x} t\left(q(t)-\lambda-\frac{2}{t}\right)\left[\frac{1}{3}\left(\frac{t^{3}}{3}\right)^{1 / 2}\|q-\lambda\|_{2}+t\right] d t\right| \\
\leq & x^{2} \int_{0}^{x}\left(\frac{t}{3}|q(t)-\lambda|+\frac{2}{3}\right)\left[\frac{1}{3}\left(\frac{t^{3}}{3}\right)^{1 / 2}\|q-\lambda\|_{2}+t\right] d t \\
\leq & x^{2}\left[\frac{1}{9} \int_{0}^{x}\left(\frac{t^{5}}{3}\right)^{1 / 2}|q(t)-\lambda|\|q-\lambda\|_{2} d t+\frac{2}{9} \int_{0}^{x}\left(\frac{t^{3}}{3}\right)^{1 / 2}\|q-\lambda\|_{2} d t\right] \\
& +x^{2}\left[\frac{1}{3} \int_{0}^{x} t^{2}|q(t)-\lambda| d t+\frac{2 t}{3}\right] .
\end{aligned}
$$

By the Cauchy-Schwarz inequality, we get

$$
\left|y_{2}(x)-y_{1}(x)\right| \leq x^{2}\left[\left(\frac{1}{3}\right)^{2}\left(\frac{x^{6}}{3.6}\right)^{1 / 2}\|q-\lambda\|_{2}^{2}+\left(\frac{x^{5}}{3.5}\right)^{1 / 2}\|q-\lambda\|_{2}+\frac{x^{2}}{2 !}\right] .
$$

Assume that the inequality is true for $k=n$. Now we will show that the inequality holds for $k=n+1$,

$$
\begin{aligned}
\left|y_{n+1}(x)-y_{n}(x)\right|= & \left|\frac{1}{3} \int_{0}^{x}\left(\frac{x^{2}}{t}-\frac{t^{2}}{x}\right)\left(q(t)-\lambda-\frac{2}{t}\right)\left(y_{n}(t)-y_{n-1}(t)\right) d t\right| \\
= & \mid \int_{0}^{x} \frac{t^{2}}{3}\left(\frac{x^{2}}{t}-\frac{t^{2}}{x}\right)\left(q(t)-\lambda-\frac{2}{t}\right)\left[\left(\frac{1}{3}\right)^{n}\left(\frac{x^{3 n}}{\prod_{m=1}^{n} 3 m}\right)^{1 / 2}\|q-\lambda\|_{2}^{n}\right. \\
& \left.+\sum_{k=1}^{n-1}\left(\frac{x^{3 n-k}}{(3 n-k) \prod_{m=1}^{n-1} 3 m}\right)^{1 / 2}\|q-\lambda\|_{2}^{n-k}+\frac{x^{n}}{n !}\right] d t \mid \\
= & \mid \int_{0}^{x}\left(\frac{t}{3}|q(t)-\lambda|+1\right)\left[\left(\frac{1}{3}\right)^{n}\left(\frac{x^{3 n}}{\prod_{m=1}^{n} 3 m}\right)^{1 / 2}\|q-\lambda\|_{2}^{n}\right. \\
& \left.+\sum_{k=1}^{n-1}\left(\frac{x^{3 n-k}}{(3 n-k) \prod_{m=1}^{n-1} 3 m}\right)^{1 / 2}\|q-\lambda\|_{2}^{n-k}+\frac{x^{n}}{n !}\right] d t \mid \\
\leq & x^{2}\left[\left(\frac{1}{3}\right)^{n+1}\left(\frac{x^{3(n+1)}}{\prod_{m=1}^{n+1} 3 m}\right)^{1 / 2}\|q-\lambda\|_{2}^{n+1}\right. \\
& \left.+\sum_{k=1}^{n}\left(\frac{x^{3(n+1)-k}}{(3(n+1)-k) \prod_{m=1}^{n} 3 m}\right)^{1 / 2}\|q-\lambda\|_{2}^{n+1-k}+\frac{x^{n+1}}{(n+1) !}\right] .
\end{aligned}
$$

By the ratio test, the series converges. Then $y_{n}(x)$ converges uniformly by the Weierstrass sufficiency theorem.

Differentiation of (2.2) gives the formula

$$
\varphi^{\prime}(x, \lambda, q)=2 x+\frac{1}{3} \int_{0}^{x}\left(\frac{2 x}{t}+\frac{t^{2}}{x^{2}}\right)\left(q(t)-\frac{2}{t}-\lambda\right) \varphi(t, \lambda, q) d t
$$

and

$$
\lim _{x \downarrow 0} x \varphi^{\prime}(x, \lambda, q)=2 .
$$


Turning to (2.3), let

$$
\begin{aligned}
& y_{0}(x)=c_{1} x^{2}+c_{2} x^{-1} \\
& y_{n+1}(x)=y_{0}(x)-\frac{1}{3} \int_{x}^{1}\left(\frac{x^{2}}{t}-\frac{t^{2}}{x}\right)\left(q(t)-\frac{2}{t}-\lambda\right) y_{n}(t) d t .
\end{aligned}
$$

Theorem 2 The sequence $x y_{n}(x)$ converges uniformly for $x \in(0,1]$ to a function $x \psi(x, \lambda, q)$ where $\psi(x, \lambda, q)$ satisfies (2.3) and (2.1). Moreover, $\lim _{x \downarrow 0} x \psi(x, \lambda, q)$ exists and the mapping $(\lambda, q) \rightarrow x \psi(x, \lambda, q)$ is analytic from $\mathbb{C} \times L^{2}[0,1] \rightarrow \mathbb{C}[0,1]$.

Proof Let us show that

$$
\begin{aligned}
\left|y_{n}(x)-y_{n-1}(x)\right| \leq & \frac{1}{x}\left[\left(\frac{1}{3}\right)^{n}\left(\frac{(1-x)^{n}}{n !}\right)^{1 / 2}\|q-\lambda\|_{2}^{n}\left|c_{n}\right|\right. \\
& \left.+\sum_{k=1}^{n-1}\left(\frac{(1-x)^{k}}{k !}\right)^{1 / 2}\|q-\lambda\|_{2}^{k}\left|c_{k}\right|+\left|c_{n+1}\right|\right]
\end{aligned}
$$

by using the method of induction. For $k=1$,

$$
\begin{aligned}
\left|y_{1}(x)-y_{0}(x)\right| & =\left|\frac{1}{3} \int_{x}^{1}\left(\frac{x^{2}}{t}-\frac{t^{2}}{x}\right)\left(q(t)-\lambda-\frac{2}{t}\right) y_{0}(t) d t\right| \\
& =\frac{1}{3} \int_{x}^{1}\left|\left(\frac{x^{2}}{t}-\frac{t^{2}}{x}\right)\left(q(t)-\lambda-\frac{2}{t}\right)\left(c_{1} t^{2}+c_{2} t^{-1}\right)\right| d t .
\end{aligned}
$$

Since $t \geq x$ we get $\left|\frac{x^{2}}{t}-\frac{t^{2}}{x}\right|=\left|\frac{t^{2}}{x}\left(\frac{x^{3}}{t^{3}}-1\right)\right| \leq \frac{t^{2}}{x}$ and

$$
\begin{aligned}
\left|y_{1}(x)-y_{0}(x)\right| & \leq \frac{1}{3} \int_{x}^{1} \frac{t^{2}}{x}\left|\left(q(t)-\lambda-\frac{2}{t}\right)\left(c_{1} t^{2}+c_{2} t^{-1}\right)\right| d t \\
& \leq \frac{1}{x}\left(\frac{1}{3} \int_{x}^{1}\left|(q(t)-\lambda)\left(c_{1} t^{4}+c_{2} t\right)\right| d t+\int_{x}^{1} \frac{2}{3}\left|c_{1} t^{3}+c_{2}\right| d t\right) .
\end{aligned}
$$

Because of $t \leq 1$, we have

$$
\left|y_{1}(x)-y_{0}(x)\right| \leq \frac{1}{x}\left(\frac{1}{3} \int_{x}^{1}|q(t)-\lambda|\left|c_{1}\right| d t+\left|c_{2}\right|\right) .
$$

By the Cauchy-Schwarz inequality, we get

$$
\left|y_{1}(x)-y_{0}(x)\right| \leq \frac{1}{x}\left((1-x)^{1 / 2}\|q-\lambda\|_{2}\left|c_{1}\right|+\left|c_{2}\right|\right) .
$$

Assume that the inequality is true for $k=n$. Now we will show that the inequality holds for $k=n+1$,

$$
\left|y_{n+1}(x)-y_{n}(x)\right|=\left|\frac{1}{3} \int_{x}^{1}\left(\frac{x^{2}}{t}-\frac{t^{2}}{x}\right)\left(q(t)-\lambda-\frac{2}{t}\right)\left(y_{n}(t)-y_{n-1}(t)\right) d t\right| \text {. }
$$


Since $t \geq x$ we get $\left|\frac{x^{2}}{t}-\frac{t^{2}}{x}\right|=\left|\frac{t^{2}}{x}\left(\frac{x^{3}}{t^{3}}-1\right)\right| \leq \frac{t^{2}}{x}$. We have

$$
\begin{aligned}
\left|y_{n+1}(x)-y_{n}(x)\right| \leq & \mid \int_{x}^{1} \frac{t^{2}}{3 x}\left(q(t)-\lambda-\frac{2}{t}\right) \frac{1}{t}\left[\left(\frac{\|q-\lambda\|}{3}\right)^{n}\left(\frac{(1-t)^{n}}{n !}\right)^{1 / 2 n}\left|c_{n}\right|\right. \\
& \left.+\sum_{k=1}^{n-1}\left(\frac{(1-t)^{k}}{k !}\right)^{1 / 2}\|q-\lambda\|_{2}^{k}\left|c_{k}\right|+\left|c_{n+1}\right|\right] d t \mid \\
\leq & \int_{x}^{1} \frac{t}{3 x}(|q(t)-\lambda|+2)\left[\left(\frac{\|q-\lambda\|}{3}\right)^{n}\left(\frac{(1-t)^{n}}{n !}\right)^{1 / 2 n}\left|c_{n}\right|\right. \\
& \left.+\sum_{k=1}^{n-1}\left(\frac{(1-t)^{k}}{k !}\right)^{1 / 2}\|q-\lambda\|_{2}^{k}\left|c_{k}\right|+\left|c_{n+1}\right|\right] d t .
\end{aligned}
$$

Because of $t \leq 1$, we have

$$
\begin{aligned}
\left|y_{n+1}(x)-y_{n}(x)\right| \leq & \frac{1}{x} \int_{x}^{1} \frac{1}{3}(|q(t)-\lambda|+2)\left[\left(\frac{\|q-\lambda\|}{3}\right)^{n}\left(\frac{(1-t)^{n}}{n !}\right)^{1 / 2 n}\left|c_{n}\right|\right. \\
& \left.+\sum_{k=1}^{n-1}\left(\frac{(1-t)^{k}}{k !}\right)^{1 / 2}\|q-\lambda\|_{2}^{k}\left|c_{k}\right|+\left|c_{n+1}\right|\right] d t .
\end{aligned}
$$

By the Cauchy-Schwarz inequality, we get

$$
\begin{aligned}
\left|y_{n+1}(x)-y_{n}(x)\right| \leq & \frac{1}{x}\left[\left(\frac{1}{3}\right)^{n+1}\left(\frac{(1-x)^{n+1}}{(n+1) !}\right)^{1 / 2}\|q-\lambda\|_{2}^{n+1}\left|c_{n+1}\right|\right. \\
& \left.+\sum_{k=1}^{n}\left(\frac{(1-x)^{k}}{k !}\right)^{1 / 2}\|q-\lambda\|_{2}^{k}\left|c_{k}\right|+\left|c_{n+2}\right|\right] .
\end{aligned}
$$

By the ratio test, the series converges. Then $y_{n}(x)$ converges uniformly by the Weierstrass sufficiency theorem.

\section{Asymptotic behavior of eigenvalues}

The main result of the paper is given by the following theorem.

Assume that $0<x_{1}<x_{2} \leq 1$.

Theorem 3 If $y$ is a nontrivial solution of the equation

$$
-y^{\prime \prime}+\left[\frac{2}{x^{2}}-\frac{2}{x}+q(x)\right] y=\lambda y
$$

with $y\left(x_{1}\right)=y^{\prime}\left(x_{2}\right)+b y\left(x_{2}\right)=0$, then

$$
\lambda \geq-\left[\frac{2}{x_{1}}+\left(|b|+2 \int_{x_{1}}^{x_{2}}|q| d x\right)^{2}\right],
$$

where $b \in \mathbb{R}$ and $q(x) \in L^{2}[0,1]$. 
Proof Multiplying (3.1) by $y$ and integrating of this equation from $x_{1}$ to $x_{2}$ gives the formula

$$
\int_{x_{1}}^{x_{2}}\left[-y^{\prime \prime} y+q(x) y^{2}-\lambda y^{2}+\left(\frac{2}{x^{2}}-\frac{2}{x}\right) y^{2}\right] d x=0 .
$$

Since $\int_{x_{1}}^{x_{2}} \frac{2}{x^{2}} y^{2} d x \geq 0$ the remaining term will be negative or zero,

$$
\int_{x_{1}}^{x_{2}}\left[-y^{\prime \prime} y+q(x) y^{2}-\lambda y^{2}-\frac{2}{x} y^{2}\right] d x \leq 0
$$

Integrating the first term by parts gives

$$
\begin{aligned}
\int_{x_{1}}^{x_{2}}-y^{\prime \prime} y d x & =-y^{\prime}\left(x_{2}\right) y\left(x_{2}\right)+y^{\prime}\left(x_{1}\right) y\left(x_{1}\right)+\int_{x_{1}}^{x_{2}}\left(y^{\prime}\right)^{2} d x \\
& =b y^{2}\left(x_{2}\right)+\int_{x_{1}}^{x_{2}}\left(y^{\prime}\right)^{2} d x .
\end{aligned}
$$

So (3.2) is equal to

$$
b y^{2}\left(x_{2}\right)+\int_{x_{1}}^{x_{2}}\left[\left(y^{\prime}\right)^{2}+q(x) y^{2}-\lambda y^{2}-\frac{2}{x} y^{2}\right] d x \leq 0 .
$$

Moreover, we find $\int_{x_{1}}^{x_{2}} q(x) y^{2}(x) d x$ and that equals

$$
\begin{aligned}
& \int_{x_{1}}^{x_{2}} 2 y(x) y^{\prime}(x) \int_{x_{1}}^{x} q(t) d t d x \\
& \quad=\left(2 y^{2}(x) \int_{x_{1}}^{x} q(t) d t\right)_{x_{1}}^{x_{2}}-\int_{x}^{x_{2}} 2 y(x) y^{\prime}(x) \int_{x_{1}}^{x} q(t) d t d x-\int_{x_{1}}^{x_{2}} 2 y^{2}(x) q(x) d x \\
& \quad=y^{2}\left(x_{2}\right) \int_{x_{1}}^{x_{2}} q(t) d t-\int_{x_{1}}^{x_{2}} y^{2}(x) q(x) d x .
\end{aligned}
$$

Then we have

$$
\int_{x_{1}}^{x_{2}} q(x) y^{2}(x) d x=y^{2}\left(x_{2}\right) \int_{x_{1}}^{x_{2}} q(t) d t-\int_{x_{1}}^{x_{2}} 2 y(x) y^{\prime}(x) \int_{x_{1}}^{x} q(t) d t d x .
$$

Integrating $\int_{x_{1}}^{x_{2}} 2 y(x) y^{\prime}(x) d x$ by parts gives

$$
\begin{aligned}
\int_{x_{1}}^{x_{2}} 2 y(x) y^{\prime}(x) d x & =\left[2 y^{2}(x)\right]_{x_{1}}^{x_{2}}-\int_{x_{1}}^{x_{2}} 2 y(x) y^{\prime}(x) d x \\
& =2 y^{2}\left(x_{2}\right)-\int_{x_{1}}^{x_{2}} 2 y(x) y^{\prime}(x) d x .
\end{aligned}
$$

So we get the formula

$$
y^{2}\left(x_{2}\right)=\int_{x_{1}}^{x_{2}} 2 y(x) y^{\prime}(x) d x
$$


Panakhov and Ulusoy Boundary Value Problems (2015) 2015:87

Page 10 of 15

Adding (3.3) to $b y^{2}\left(x_{2}\right)$ and using the triangle inequality gives the formula

$$
\begin{aligned}
& \left|b y^{2}\left(x_{2}\right)\right|+\left|\int_{x_{1}}^{x_{2}} q(x) y^{2}(x) d x\right| \\
& \leq|b| y^{2}\left(x_{2}\right)+\left|y^{2}\left(x_{2}\right) \int_{x_{1}}^{x_{2}} q(t) d t\right|+\left|\int_{x_{1}}^{x_{2}} 2 y(x) y^{\prime}(x) \int_{x_{1}}^{x} q(t) d t d x\right| \\
& \quad \leq|b| \int_{x_{1}}^{x_{2}} 2 y y^{\prime} d x+\int_{x_{1}}^{x_{2}}|q| d x \int_{x_{1}}^{x_{2}} 2 y y^{\prime} d x+\int_{x_{1}}^{x_{2}}\left|2 y y^{\prime}\right| \int_{x_{1}}^{x}|q(t)| d t d x .
\end{aligned}
$$

Since $x \leq x_{2}$ we get

$$
\left|b y^{2}\left(x_{2}\right)\right|+\left|\int_{x_{1}}^{x_{2}} q(x) y^{2}(x) d x\right| \leq\left[|b|+2 \int_{x_{1}}^{x_{2}}|q| d x\right] \int_{x_{1}}^{x_{2}} 2 y y^{\prime} d x .
$$

By the Cauchy-Schwarz inequality, we get

$$
\begin{aligned}
& \left|b y^{2}\left(x_{2}\right)\right|+\left|\int_{x_{1}}^{x_{2}} q(x) y^{2}(x) d x\right| \\
& \quad \leq\left(|b|+2 \int_{x_{1}}^{x_{2}}|q| d x\right) 2\left[\int_{x_{1}}^{x_{2}} y^{2} d x\right]^{1 / 2}\left[\int_{x_{1}}^{x_{2}}\left(y^{\prime}\right)^{2} d x\right]^{1 / 2}
\end{aligned}
$$

and, from the inequality of $2 A^{1 / 2} B^{1 / 2} \leq \varepsilon A+(1 / \varepsilon) B$, we get

$$
\begin{aligned}
& \left|b y^{2}\left(x_{2}\right)\right|+\left|\int_{x_{1}}^{x_{2}} q(x) y^{2}(x) d x\right| \\
& \quad \leq\left(|b|+2 \int_{x_{1}}^{x_{2}}|q| d x\right)\left[\varepsilon\left(\int_{x_{1}}^{x_{2}} y^{2} d x\right)+1 / \varepsilon\left(\int_{x_{1}}^{x_{2}}\left(y^{\prime}\right)^{2} d x\right)\right] .
\end{aligned}
$$

For any $\varepsilon>|b|+2 \int_{x_{1}}^{x_{2}}|q| d x$,

$$
\begin{aligned}
& b y^{2}\left(x_{2}\right)+\int_{x_{1}}^{x_{2}}\left[\left(y^{\prime}\right)^{2}+q(x) y^{2}-\lambda y^{2}-\frac{2}{x} y^{2}\right] d x \\
& \geq \int_{x_{1}}^{x_{2}}\left[\left(y^{\prime}\right)^{2}-\lambda y^{2}-\frac{2}{x} y^{2}\right] d x-|b| y^{2}\left(x_{2}\right)-\left|\int_{x_{1}}^{x_{2}} q y^{2} d x\right| \\
& \geq \int_{x_{1}}^{x_{2}}\left[\left(y^{\prime}\right)^{2}-\lambda y^{2}\right] d x-\frac{2}{x_{1}} \int_{x_{1}}^{x_{2}} y^{2} d x \\
&-\left(|b|+2 \int_{x_{1}}^{x_{2}}|q| d x\right)\left[\varepsilon\left(\int_{x_{1}}^{x_{2}} y^{2} d x\right)+1 / \varepsilon\left(\int_{x_{1}}^{x_{2}}\left(y^{\prime}\right)^{2} d x\right)\right] \\
& \geq {\left[1-\left(|b|+2 \int_{x_{1}}^{x_{2}}|q| d x\right) / \varepsilon\right] \int_{x_{1}}^{x_{2}}\left(y^{\prime}\right)^{2} d x } \\
&+\left[-\lambda-\frac{2}{x_{1}}-\varepsilon\left(|b|+2 \int_{x_{1}}^{x_{2}}|q| d x\right)\right] \int_{x_{1}}^{x_{2}} y^{2} d x \\
&= C(\varepsilon)+\left[-\lambda-\frac{2}{x_{1}}-\varepsilon\left(|b|+2 \int_{x_{1}}^{x_{2}}|q| d x\right)\right] \int_{x_{1}}^{x_{2}} y^{2} d x,
\end{aligned}
$$


where $C(\varepsilon)>0$. If we assume that

$$
\lambda<-\left[\frac{2}{x_{1}}+\left(|b|+2 \int_{x_{1}}^{x_{2}}|q| d x\right)^{2}\right]
$$

the equation $\int_{x_{1}}^{x_{2}}\left[-y^{\prime \prime} y+q(x) y^{2}-\lambda y^{2}-\frac{2}{x} y^{2}\right] d x$ should be positive. It contradicts our assumption. So we proved the theorem.

Theorem 4 If $y$ is a nontrivial solution of the equation

$$
-y^{\prime \prime}+\left[\frac{2}{x^{2}}-\frac{2}{x}+q(x)\right] y=\lambda y
$$

with $y\left(x_{1}\right)=y\left(x_{2}\right)=0$, then

$$
\lambda \geq-\left[\frac{2}{x_{1}}+4\left(\int_{x_{1}}^{x_{2}}|q| d x\right)^{2}\right]
$$

where $q(x) \in L^{2}[0,1]$.

Proof Multiplying the equation by $y$ and integrating of this equation from $x_{1}$ to $x_{2}$ gives the formula

$$
\int_{x_{1}}^{x_{2}}\left[-y^{\prime \prime} y+q(x) y^{2}-\lambda y^{2}+\left(\frac{2}{x^{2}}-\frac{2}{x}\right) y^{2}\right] d x=0 .
$$

Since $\int_{x_{1}}^{x_{2}} \frac{2}{x^{2}} y^{2} d x \geq 0$ the remaining term will be negative or zero,

$$
\int_{x_{1}}^{x_{2}}\left[-y^{\prime \prime} y+q(x) y^{2}-\lambda y^{2}-\frac{2}{x} y^{2}\right] d x \leq 0 .
$$

Integrating the first term by parts gives

$$
\int_{x_{1}}^{x_{2}}-y^{\prime \prime} y d x=-y^{\prime}\left(x_{2}\right) y\left(x_{2}\right)+y^{\prime}\left(x_{1}\right) y\left(x_{1}\right)+\int_{x_{1}}^{x_{2}}\left(y^{\prime}\right)^{2} d x=\int_{x_{1}}^{x_{2}}\left(y^{\prime}\right)^{2} d x .
$$

So (3.4) is equal to

$$
b y^{2}\left(x_{2}\right)+\int_{x_{1}}^{x_{2}}\left[\left(y^{\prime}\right)^{2}+q(x) y^{2}-\lambda y^{2}-\frac{2}{x} y^{2}\right] d x \leq 0 .
$$

Moreover, we find $\int_{x_{1}}^{x_{2}} q(x) y^{2}(x) d x$ and that equals

$$
\begin{aligned}
& \int_{x_{1}}^{x_{2}} 2 y(x) y^{\prime}(x) \int_{x_{1}}^{x} q(t) d t d x \\
& \quad=\left(2 y^{2}(x) \int_{x_{1}}^{x} q(t) d t\right)_{x_{1}}^{x_{2}}-\int_{x}^{x_{2}} 2 y(x) y^{\prime}(x) \int_{x_{1}}^{x} q(t) d t d x-\int_{x_{1}}^{x_{2}} 2 y^{2}(x) q(x) d x \\
& \quad=-\int_{x_{1}}^{x_{2}} y^{2}(x) q(x) d x .
\end{aligned}
$$


Then we have

$$
\int_{x_{1}}^{x_{2}} q(x) y^{2}(x) d x=-\int_{x_{1}}^{x_{2}} 2 y(x) y^{\prime}(x) \int_{x_{1}}^{x} q(t) d t d x
$$

since $x \leq x_{2}$ we get

$$
\left|\int_{x_{1}}^{x_{2}} q(x) y^{2}(x) d x\right| \leq \int_{x_{1}}^{x_{2}}|q| d x\left|\int_{x_{1}}^{x_{2}} 2 y y^{\prime} d x\right| .
$$

By the Cauchy-Schwarz inequality, we get

$$
\left|\int_{x_{1}}^{x_{2}} q(x) y^{2}(x) d x\right| \leq 2 \int_{x_{1}}^{x_{2}}|q| d x\left[\int_{x_{1}}^{x_{2}} y^{2} d x\right]^{1 / 2}\left[\int_{x_{1}}^{x_{2}}\left(y^{\prime}\right)^{2} d x\right]^{1 / 2},
$$

and from the inequality of $2 A^{1 / 2} B^{1 / 2} \leq \varepsilon A+(1 / \varepsilon) B$, we get

$$
\left|\int_{x_{1}}^{x_{2}} q(x) y^{2}(x) d x\right| \leq 2 \int_{x_{1}}^{x_{2}}|q| d x\left[\varepsilon\left(\int_{x_{1}}^{x_{2}} y^{2} d x\right)+1 / \varepsilon\left(\int_{x_{1}}^{x_{2}}\left(y^{\prime}\right)^{2} d x\right)\right] .
$$

For any $\varepsilon>2 \int_{x_{1}}^{x_{2}}|q| d x$,

$$
\begin{aligned}
\int_{x_{1}}^{x_{2}} & {\left[\left(y^{\prime}\right)^{2}+q(x) y^{2}-\lambda y^{2}-\frac{2}{x} y^{2}\right] d x } \\
\geq & \int_{x_{1}}^{x_{2}}\left[\left(y^{\prime}\right)^{2}-\lambda y^{2}-\frac{2}{x} y^{2}\right] d x-\left|\int_{x_{1}}^{x_{2}} q y^{2} d x\right| \\
\geq & \int_{x_{1}}^{x_{2}}\left[\left(y^{\prime}\right)^{2}-\lambda y^{2}\right] d x-\frac{2}{x_{1}} \int_{x_{1}}^{x_{2}} y^{2} d x \\
& -2 \int_{x_{1}}^{x_{2}}|q| d x\left[\varepsilon\left(\int_{x_{1}}^{x_{2}} y^{2} d x\right)+1 / \varepsilon\left(\int_{x_{1}}^{x_{2}}\left(y^{\prime}\right)^{2} d x\right)\right] \\
\geq & {\left[1-\frac{2}{\varepsilon} \int_{x_{1}}^{x_{2}}|q| d x\right] \int_{x_{1}}^{x_{2}}\left(y^{\prime}\right)^{2} d x } \\
& +\left[-\lambda-\frac{2}{x_{1}}-2 \varepsilon \int_{x_{1}}^{x_{2}}|q| d x\right] \int_{x_{1}}^{x_{2}} y^{2} d x \\
= & C(\varepsilon)+\left[-\lambda-\frac{2}{x_{1}}-\varepsilon\left(|b|+2 \int_{x_{1}}^{x_{2}}|q| d x\right)\right] \int_{x_{1}}^{x_{2}} y^{2} d x,
\end{aligned}
$$

where $C(\varepsilon)>0$. If we assume that

$$
\lambda<-\left[\frac{2}{x_{1}}+4\left(\int_{x_{1}}^{x_{2}}|q| d x\right)^{2}\right],
$$

the equation $\int_{x_{1}}^{x_{2}}\left[-y^{\prime \prime} y+q(x) y^{2}-\lambda y^{2}-\frac{2}{x} y^{2}\right] d x$ should be positive. It contradicts our assumption. So we get our result.

Theorem 5 If $y$ is a nontrivial solution of the equation

$$
-y^{\prime \prime}+\left[\frac{2}{x^{2}}-\frac{2}{x}+q(x)\right] y=0
$$


where $q(x) \in L^{2}[0,1]$, and if $y^{\prime}\left(x_{1}\right)=y^{\prime}\left(x_{2}\right)=0$, where $0 \leq x_{1} \leq x_{2} \leq 1$, then

$$
\frac{1}{x_{2}^{2}}+\frac{1}{x_{2}} \leq 2\left[\int_{x_{1}}^{x_{2}}|q| d x\right]^{2}+\frac{1}{2\left(x_{2}-x_{1}\right)} \int_{x_{1}}^{x_{2}}|q| d x .
$$

Proof Multiplying (3.5) by $y$ and integrating of this equation from $x_{1}$ to $x_{2}$ gives the formula

$$
\int_{x_{1}}^{x_{2}}\left[-y^{\prime \prime} y+q(x) y^{2}+\left(\frac{2}{x^{2}}-\frac{2}{x}\right) y^{2}\right] d x=0
$$

Since

$$
\int_{x_{1}}^{x_{2}} 2 y(x) y^{\prime}(x) \int_{x_{1}}^{x} q(t) d t d x=y^{2}\left(x_{2}\right) \int_{x_{1}}^{x_{2}} q(t) d t-\int_{x_{1}}^{x_{2}} q(x) y^{2}(x) d x
$$

we get

$$
\int_{x_{1}}^{x_{2}} q(x) y^{2}(x) d x=y^{2}\left(x_{2}\right) \int_{x_{1}}^{x_{2}} q(t) d t-\int_{x_{1}}^{x_{2}} 2 y(x) y^{\prime}(x) \int_{x_{1}}^{x} q(t) d t d x .
$$

From the mean value theorem we can write

$$
y^{2}\left(x_{3}\right)=\left[1 /\left(x_{2}-x_{1}\right)\right] \int_{x_{1}}^{x_{2}} y^{2} d x
$$

where $x_{3} \in\left[x_{1}, x_{2}\right]$. From (3.7) and integrating $\int_{x_{1}}^{x_{2}} 2 y(x) y^{\prime}(x) d x$ by parts we have

$$
y^{2}\left(x_{2}\right)=y^{2}\left(x_{3}\right)+\int_{x_{1}}^{x_{2}} 2 y y^{\prime} d x=\frac{1}{x_{2}-x_{1}} \int_{x_{1}}^{x_{2}} y^{2} d x+\int_{x_{1}}^{x_{2}} 2 y y^{\prime} d x .
$$

Adding (3.6) to $\int_{x_{1}}^{x_{2}} \frac{2 y^{2}}{x} d x$ we have

$$
\begin{aligned}
\int_{x_{1}}^{x_{2}}\left(q-\frac{2}{x}\right) y^{2} d x= & {\left[\frac{1}{x_{2}-x_{1}} \int_{x_{1}}^{x_{2}} y^{2} d x+\int_{x_{1}}^{x_{2}} 2 y y^{\prime} d x\right] \int_{x_{1}}^{x_{2}} q d x } \\
& -\int_{x_{1}}^{x_{2}} 2 y y^{\prime} \int_{x_{1}}^{x} q(t) d t d x-\int_{x_{1}}^{x_{2}} \frac{2 y^{2}}{x} d x .
\end{aligned}
$$

From the triangle inequality we have the formula

$$
\begin{aligned}
\left|\int_{x_{1}}^{x_{2}}\left(q-\frac{2}{x}\right) y^{2} d x\right| \leq & \left|\left[\frac{1}{x_{2}-x_{1}} \int_{x_{1}}^{x_{2}} y^{2} d x+\int_{x_{1}}^{x_{2}} 2 y y^{\prime} d x\right] \int_{x_{1}}^{x_{2}} q d x\right| \\
& +\left|\int_{x_{1}}^{x_{2}} 2 y y^{\prime} \int_{x_{1}}^{x} q d t d x\right|+\left|\int_{x_{1}}^{x_{2}} \frac{2 y^{2}}{x} d x\right| \\
\leq & \left(\frac{1}{x_{2}-x_{1}} \int_{x_{1}}^{x_{2}}|q| d x+\frac{2}{x_{2}}\right) \int_{x_{1}}^{x_{2}} y^{2} d x+2 \int_{x_{1}}^{x_{2}} 2\left|y y^{\prime}\right| \int_{x_{1}}^{x} q d t d x .
\end{aligned}
$$

By using the inequality

$$
2\left|y y^{\prime}\right| \leq \varepsilon y^{2}+(1 / \varepsilon)\left(y^{\prime}\right)^{2},
$$


Panakhov and Ulusoy Boundary Value Problems (2015) 2015:87

Page 14 of 15

we get

$$
\begin{aligned}
\left|\int_{x_{1}}^{x_{2}}\left(q-\frac{2}{x}\right) y^{2} d x\right| \leq & {\left[\frac{1}{x_{2}-x_{1}} \int_{x_{1}}^{x_{2}}|q| d x+\frac{2}{x_{2}}\right] \int_{x_{1}}^{x_{2}} y^{2} d x } \\
& +2\left[\varepsilon \int_{x_{1}}^{x_{2}} y^{2} d x+\frac{1}{\varepsilon} \int_{x_{1}}^{x_{2}}\left(y^{\prime}\right)^{2} d x\right] \int_{x_{1}}^{x_{2}}|q| d x .
\end{aligned}
$$

Integrating $\int_{x_{1}}^{x_{2}}-y^{\prime \prime} y d x$ by parts gives

$$
\begin{aligned}
& \int_{x_{1}}^{x_{2}}-y^{\prime \prime} y d x=-y^{\prime}\left(x_{2}\right) y\left(x_{2}\right)+y^{\prime}\left(x_{1}\right) y\left(x_{1}\right)+\int_{x_{1}}^{x_{2}}\left(y^{\prime}\right)^{2} d x=\int_{x_{1}}^{x_{2}}\left(y^{\prime}\right)^{2} d x, \\
& \int_{x_{1}}^{x_{2}} \frac{2}{x^{2}} y^{2} d x \geq \int_{x_{1}}^{x_{2}} \frac{2}{x_{2}^{2}} y^{2} d x .
\end{aligned}
$$

From (3.9) and (3.8) and for any $\varepsilon>|b|+2 \int_{x_{1}}^{x_{2}}|q| d x$, there exists a number $C(\varepsilon)>0$ such that

$$
\begin{aligned}
& \int_{x_{1}}^{x_{2}}\left[-y^{\prime \prime} y+q(x) y^{2}+\left(\frac{2}{x^{2}}-\frac{2}{x}\right) y^{2}\right] d x \\
&=\int_{x_{1}}^{x_{2}}\left(y^{\prime}\right)^{2} d x+\int_{x_{1}}^{x_{2}}\left(q-\frac{2}{x}\right) y^{2} d x+\int_{x_{1}}^{x_{2}} \frac{2}{x^{2}} y^{2} d x \\
& \geq \int_{x_{1}}^{x_{2}}\left(y^{\prime}\right)^{2} d x-\left(\frac{1}{x_{2}-x_{1}} \int_{x_{1}}^{x_{2}}|q| d x+\frac{2}{x_{2}}\right) \int_{x_{1}}^{x_{2}} y^{2} d x \\
&-2\left(\varepsilon \int_{x_{1}}^{x_{2}} y^{2} d x+\frac{1}{\varepsilon} \int_{x_{1}}^{x_{2}}\left(y^{\prime}\right)^{2} d x\right) \int_{x_{1}}^{x_{2}}|q| d x+\int_{x_{1}}^{x_{2}} \frac{2}{x_{2}^{2}} y^{2} d x \\
&=\left(1-\frac{2}{\varepsilon} \int_{x_{1}}^{x_{2}}|q| d x\right) \int_{x_{1}}^{x_{2}}\left(y^{\prime}\right)^{2} d x \\
&+\left\{\frac{2}{x_{2}^{2}}+\frac{2}{x_{2}}-\left(\frac{1}{x_{2}-x_{1}}+2 \varepsilon\right) \int_{x_{1}}^{x_{2}}|q| d x\right\} \int_{x_{1}}^{x_{2}} y^{2} d x \\
&= C(\varepsilon)+\left\{\frac{2}{x_{2}^{2}}+\frac{2}{x_{2}}-\left(\frac{1}{x_{2}-x_{1}}+2 \varepsilon\right) \int_{x_{1}}^{x_{2}}|q| d x\right\} \int_{x_{1}}^{x_{2}} y^{2} d x .
\end{aligned}
$$

Let us assume that

$$
\frac{2}{x_{2}^{2}}+\frac{2}{x_{2}}-\left(\frac{1}{x_{2}-x_{1}}+2 \varepsilon\right) \int_{x_{1}}^{x_{2}}|q| d x>0 .
$$

In this case, we get

$$
\begin{aligned}
\frac{1}{x_{2}^{2}}+\frac{1}{x_{2}} & >\varepsilon \int_{x_{1}}^{x_{2}}|q| d x+\frac{1}{2\left(x_{2}-x_{1}\right)} \int_{x_{1}}^{x_{2}}|q| d x \\
& >2\left(\int_{x_{1}}^{x_{2}}|q| d x\right)^{2}+\frac{1}{2\left(x_{2}-x_{1}\right)} \int_{x_{1}}^{x_{2}}|q| d x .
\end{aligned}
$$

Then we have

$$
\int_{x_{1}}^{x_{2}}\left[-y^{\prime \prime} y+q(x) y^{2}+\left(\frac{2}{x^{2}}-\frac{2}{x}\right) y^{2}\right] d x>0 .
$$


This is a contradiction. So we proved the theorem.

Conclusion In the Carlson case, the potentials are in $L^{2}[0,1]$, but in our paper, the potentials are not in $L^{2}[0,1]$.

\section{Competing interests}

The authors declare that they have no competing interests.

\section{Authors' contributions}

IU carried out the design of the study and performed the analysis. EP (adviser) participated in its design and coordination All authors read and approved the final manuscript.

\section{Author details}

${ }^{1}$ Department of Mathematics, Firat University, Elazig, 23119, Turkey. ${ }^{2}$ Department of Mathematics, Adiyaman University, Adiyaman, 02040, Turkey.

\section{Acknowledgements}

The authors would like to thank the referees for valuable comments in improving the original paper.

Received: 23 November 2014 Accepted: 11 May 2015 Published online: 02 June 2015

\section{References}

1. Birkhoff, GD: Boundary value and expansion problems of ordinary linear differential equations. Trans. Am. Math. Soc. 9(4), 373-395 (1908)

2. Titchmarsh, EC: Eigenfunction Expansions Associated with Second-Order Differential Equations, vol. 1. Clarendon, Oxford (1946)

3. Gasymov, MG: The definition of Sturm-Liouville operator from two spectra. DAN SSSR 161(2), 274-276 (1965)

4. Levitan, BM, Sargsyan, IS: Introduction to Spectral Theory: Selfadjoint Ordinary Differential Operators. Am. Math. Soc., Providence (1975)

5. Courant, R, Hilbert, D: Methods of Mathematical Physics, vol. I. Interscience, New York (1953)

6. Birman, MS, Solomyak, MZ: On asymptotic behaviour of the spectrum of variational problems with constraints. In: Proceedings of the Conference Dedicated to Academician Petrovsky 75th Birthday, pp. 51-52. Izdat. Mosk. Univ., Moscow (1978) (Russian)

7. Carleman, T: Problèmes Mathématiques dans la Théorie Cinétique des Gaz. Almqvist och Wiksells Boktryckeri, Uppsala (1957) (in French)

8. Fedoryuk, MV: Asymptotic Methods in Analysis. Encyclopaedia of Mathematical Sciences, vol. 13, pp. 83-191 (1989)

9. Poeschel, J, Trubowitz, E: Inverse Spectral Theory. Academic Press, San Diego (1987)

10. Carlson, R: Inverse spectral theory for some singular Sturm-Liouville problems. J. Differ. Equ. 106, 121-140 (1993)

11. Hochstadt, H, Lieberman, B: An inverse Sturm-Liouville problem with mixed given data. SIAM J. Appl. Math. 34 676-680 (1978)

12. Panakhov, ES, Yilmazer, R: A Hochstadt-Lieberman theorem for the hydrogen atom equation. Appl. Comput. Math. 11(1), 74-80 (2012)

13. Panakhov, ES, Yilmazer, R: On inverse problem for singular Sturm-Liouville operator from two spectra. Ukr. Math. J. 58(1), 147-154 (2006)

14. Koyunbakan, H, Panakhov, ES: Half inverse problem for singular differential operator. Appl. Anal. 84(3), 247-252 (2005)

15. Panakhov, ES, Sat, M: Reconstruction of potential function for Sturm-Liouville operator with Coulomb potential. Bound. Value Probl. 2013, 49 (2013)

16. Guillot, J-C, Ralston, JV: Inverse spectral theory for a singular Sturm-Liouville operator on [0, 1]. J. Differ. Equ. 76 , 353-373 (1988)

17. Zhornitskaya, LA, Serov, VS: Inverse eigenvalue problems for a singular Sturm-Liouville operator on $[0,1]$. Inverse Probl. 10(4), 975-987 (1994)

18. Serier, F: The inverse spectral problem for radial Schrödinger operators on [0, 1]. J. Differ. Equ. 235, $101-126$ (2007)

19. Bas, E, Metin, F: Fractional singular Sturm-Liouville operator for Coulomb potential. Adv. Differ. Equ. 2013,300 (2013)

20. Newton, RG: Scattering Theory of Waves and Particles. Texts and Monographs in Physics. Springer, New York (1982)

21. Kostyuchenko, AG, Sargsyan, IS: Distribution of Eigenvalues. Nauka, Moscow (1979)

22. Rundell, W, Sacks, PE: Reconstruction techniques for classical inverse Sturm-Liouville problems. Math. Comput. 58(197), 161-183 (1992)

23. Clark, C: The asymptotic distribution of eigenvalues and eigenfunctions for elliptic boundary value problems. SIAM Rev. 9, 627-646 (1967)

24. Olver, FWJ: Introduction to Asymptotics and Special Functions. Academic Press, New York (1974)

25. de Wet, JS, Mandl, F: On the asymptotic distribution of eigenvalues. Proc. R. Soc. Lond. A 200, $572-580$ (1950)

26. Morse, P, Feshbach, H: Methods of Theoretical Physics. McGraw-Hill, London (1953)

27. Blohincev, Dl: Foundations of Quantum Mechanics. GITTL, Moscow (1949) (3rd edn.: Vyss. Skola, Kiev (1961); English transl.: Reidel, Dordrecht (1964))

28. Fok, VA: Beginnings of Quantum Mechanics. Izdat. Leningrad Gos. Univ., Leningrad (1932) 